\title{
A possible transport mechanism for aluminum in biological membranes
}

\author{
Marcantonio Bragadin ${ }^{\mathrm{a}, *}$, Sabrina Manente ${ }^{\mathrm{a}}$, Guido Scutari ${ }^{\mathrm{b}}$, Maria Pia Rigobello ${ }^{\mathrm{b}}$, \\ Alberto Bindoli ${ }^{b}$ \\ a Dipartimento di Scienze Ambientali, Università di Venezia, DD 2137, 30123 Venezia, Italy \\ ${ }^{\mathrm{b}}$ Dipartimento di Chimica Biologica e centro Studio delle Biomembrane del C.N.R., Università di Padova, Viale G. Colombo 3, 35121 Padova, Italy
}

Received 18 June 2003; received in revised form 24 February 2004; accepted 12 March 2004

Available online 22 April 2004

\begin{abstract}
The transport mechanism of aluminum in lysosomes extracted from rat liver has been investigated in this paper. The experimental evidence supports the hypothesis that aluminum is transported inside lysosomes in the form of an $\mathrm{Al}(\mathrm{OH})_{3}$ electroneutral compound, the driving force being the internal acidic $\mathrm{pH}$. This mechanism could help to explain the presence of aluminum in cells in many illnesses.
\end{abstract}

(C) 2004 Elsevier Inc. All rights reserved.

Keywords: Aluminum; Lysosomes; Transport

The toxicity of aluminum and, in particular, its neurotoxicity has already been widely documented in both humans and animals [1-7]. In order to study the molecular mechanism responsible for the toxic effects of this metal on whole organisms, many "in vitro" investigations have already been carried out [8-12].

The results indicate that aluminum has been found inside the cell in many illnesses. Many models and transport mechanisms have already been proposed in an attempt to explain the transport mechanism which allows the aluminum to permeate the cell. It has been proposed that, in some cases, the aluminum binds to certain natural carriers such as the catecholamines $[13,14]$, transferrin [15] or, simply, to the aminoacids [16]. In this paper, we have examined the transport mechanism of aluminum in lysosomes extracted from rat liver, as this mechanism is a crucial starting point regarding the study of the molecular toxicity mechanism. The results indicate that aluminum enters the matrix as an $\mathrm{Al}(\mathrm{OH})_{3}$ electroneutral compound. The

\footnotetext{
${ }^{*}$ Corresponding author. Tel.: +39-41-234-8507; fax: +39-41-2348584.

E-mail address: bragadin@unive.it (M. Bragadin).
}

behaviour displayed in this case is similar to that displayed by the weak permeant bases.

In non-energized lysosomes, the $\mathrm{pH}$ in the matrix is about 5.2, resulting from the presence of a Donnan effect [19]. The addition of ATP to the resuspending medium produces a further acidification as a consequence of the presence of an ATP-driven proton pump (Fig. 1) [20-23]. However, since the lysosomal membrane is not permeant to the protons, and since the influx of protons gives rise to a positive-inside potential, the acidification is not significant, i.e. the positive-inside potential is an opposing force, which does not allow for an acidification of less than $\mathrm{pH} 5$. In the presence of the $\mathrm{Cl}^{-}$ions in the resuspending medium, a selective $\mathrm{Cl}^{-}$channel allows for the transport of the $\mathrm{Cl}^{-}$into the matrix, and the subsequent uptake of the $\mathrm{Cl}^{-}$partially neutralizes the positive-inside potential. Under these conditions the $\mathrm{pH}$ reaches a value of about 4.2 [20-23]. The $\mathrm{pH}$ is measured using the acridine orange (AO) dye. AO is a weak base which, following the uptake mechanism of weak permeant bases accumulates in the matrix if the $\mathrm{pH}$ is acidic $[21,24]$. This accumulation is accompanied by a chemical shift [23] probably due to a metachromatic effect [25]. Therefore, by operating at $492 \mathrm{~nm}$, which is the 


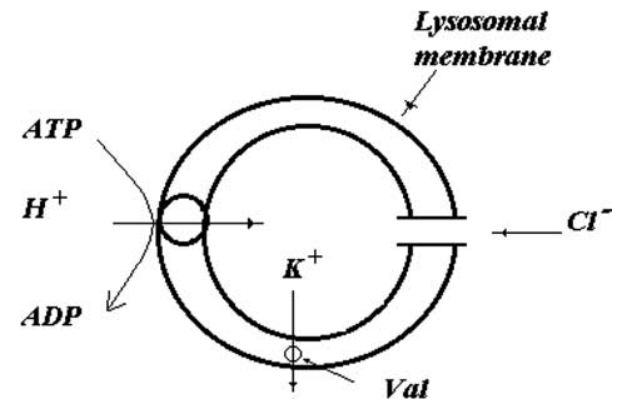

Fig. 1. Acidification in lysosomes. In the presence of ATP, the ATPdriven proton pump pumps the protons into the matrix and the $\mathrm{pH}$ decreases to a level of 4.2, providing the influx of chloride ions by means of a selective channel (or the $\mathrm{K}^{+}$efflux, in the presence of Valinomycin (Val), in a chloride-free medium).

maximum absorbance of $\mathrm{AO}$ in water, a decrease in the absorbance indicates the accumulation of $\mathrm{AO}$ in the matrix, and is a measure of the internal $\mathrm{pH}$, since the $\mathrm{pH}$ is the driving force for the accumulation of weak permeant bases. A typical experiment in which the internal $\mathrm{pH}$ is measured by means of the AO signal is illustrated in Fig. 2. Before the addition of the ATP, the value of the absorbance in a steady state is correlated with the internal $\mathrm{pH}$, which is about 5.2, as above mentioned. After the ATP addition, a slow acidification occurs and, after some minutes, the new steady state corresponds to an internal $\mathrm{pH}$ of about 4.2. Under these conditions, the addition of $\mathrm{Al}$ (as $\mathrm{Al}(\text { lactate) })_{3}$ ) induces a rapid internal alkalinization (Fig. 2).

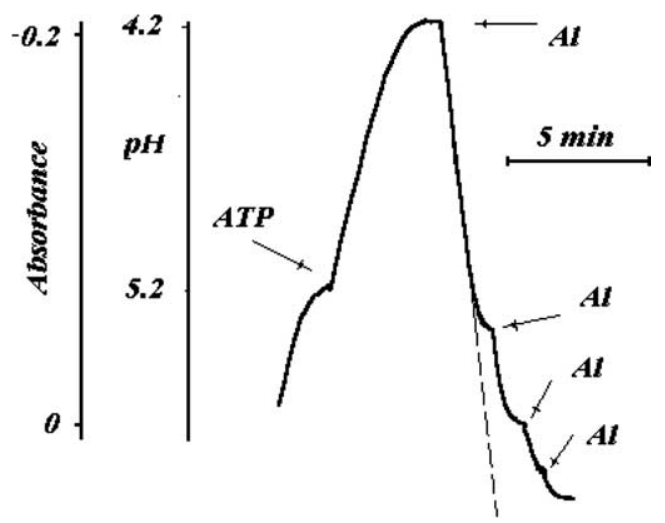

Fig. 2. The acidification of lysosomes measured using the acridine orange (AO) dye. The lysosomes were prepared from rat liver, purified by means of the usual procedures [17], and resuspended in a medium containing the following: $125 \mathrm{mM} \mathrm{KCl}, 10 \mathrm{mM}$ Hepes-Mops $\mathrm{pH}$ 7.4, $10 \mathrm{mM} \mathrm{MgCl} 2,5 \mu \mathrm{M} \mathrm{AO}$. After the addition of the Lysosomes (final concentration $0.2 \mathrm{mg} / \mathrm{ml}$ ) to the medium, the instrument was adjusted to zero absorbance. The concentration of Lysosomes was monitored by using the Lowry procedure [18]. The absorbance decrease at $492 \mathrm{~nm}$ is a measure of the internal $\mathrm{pH}(5.2)$ before ATP $(2 \mathrm{mM})$ addition. Successive additions of $80 \mu \mathrm{M} \mathrm{Al}$ (final concentration of aluminum, in the form of lactate, in the resuspending medium) in a steady state, after the ATP addition, induce a rapid alkalinization in the lysosomes, while the external $\mathrm{pH}$ (7.4), was not modified. The slope of the dashed line indicates the rate of alkalinization induced by the aluminum.
In general, this alkalinization process can occur by means of four different mechanisms:

- Inhibition of the proton pump. Fig. 3 shows the results of experiments performed in order to verify if the proton pump (or vacuolar ATPase) is inhibited by aluminum. The graphic shows that aluminum really induces an inhibition of the ATP hydrolysis rate. Analogously, the effect of the inhibition of the pump can be followed measuring the alkalinization rate in lysosomes as consequence of the addition of Bafilomycin a potent ATPase inhibitor, in energized lysosomes (Fig. 4) [26]. A comparison between the two alkalinization rates obtained with the addition of alu-

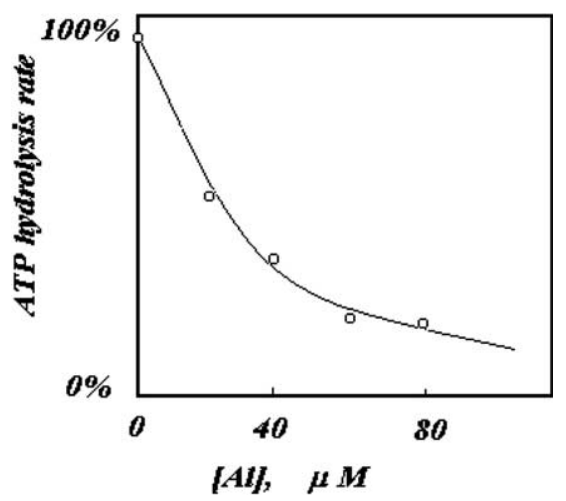

Fig. 3. Aluminum inhibits the proton pump. The inhibition of the ATPase has been followed in a low buffered medium following the $\mathrm{pH}$ change induced by ATP hydrolysis: ATP $\rightarrow$ ADP + phosphate $+\mathrm{H}^{+}$ medium composition: $125 \mathrm{mM} \mathrm{KCl}, 50 \mu \mathrm{M}$ Hepes-Mops $\mathrm{pH} 7.4,10$ $\mathrm{mM} \mathrm{MgCl} 2$. To the medium (4 ml) were added (under stirring) Oligomycin $2 \gamma$, FCCP $100 \mathrm{nM}$, the lysosomes (final concentration $0.2 \mathrm{mg}$ / $\mathrm{ml}$ ) and ATP $1 \mathrm{mM}$. The acidification rate induced by ATP is measured in the presence of increasing amounts of aluminum. The hydrolysis rates are reported in the graphic as percentage of inhibition between the two extreme rates: in the absence of aluminum $(100 \%)$ and in the presence of bafilomycin (rate $0 \%$ ).

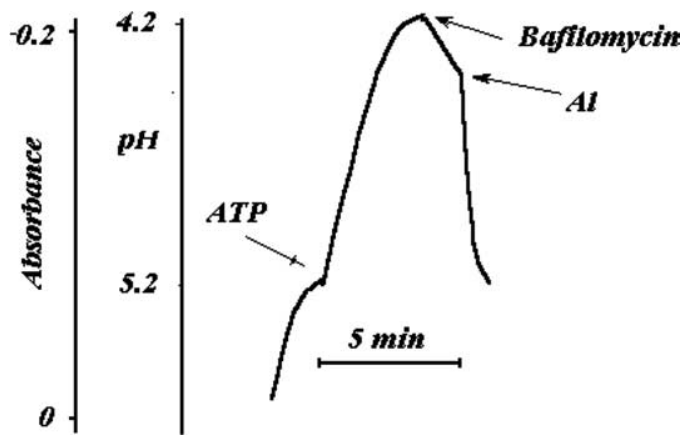

Fig. 4. Bafilomycin induces inhibition of the lysosomal pump. Both the medium used and the conditions were the same as those in Fig. 2. 50 $\mathrm{nM}$ bafilomycin were added in steady state (a). The rate of alkalinization induced by bafilomycin is represented by the slope of the dashed line. The addition of $80 \mu \mathrm{M}$ aluminum (in the form of lactate) gives rise to a further increase of the alkalinization rate (b). The rate of alkalinization with bafilomycin depends on the proton leak in lysosomes. This value of this rate can depend on the preparation of the lysosomes and on their aging, but in all cases, the addition of aluminum induces a fast increase of the alkalinization rate. 
minum (Fig. 2) or using bafilomycin (Fig. 4), makes it clear that aluminum, if added in energized conditions, even if it inhibits the ATPase (Fig. 3), acts differently from a pure inhibitor of the ATPase (bafilomycin). This interpretation is supported by means of the addition of aluminum after Bafilomycin (Fig. 4). Under this condition (i.e. inhibition of the proton pump by means of bafilomycin), the addition of aluminum causes a further enhancement of the alkalinization rate, thus indicating that the effect of aluminum is different from the one induced by means of bafilomycin and that the rapid alkalinization rate observed in Fig. 2 is not the consequence of the inhibition of the ATPase by aluminum.

- Inhibition of the chloride channel. As a means of investigating this possibility, the alkalinization of the lysosomes was performed using a chloride-free medium. For the purposes of this experiment (Fig. 5), the medium contained $80 \mathrm{mM} \mathrm{K}_{2} \mathrm{SO}_{4}$. The internal acidification which took place after the ATP addition was not a consequence of the $\mathrm{Cl}^{-}$influx, but of the $\mathrm{K}^{+}$efflux coming from the lysosomal matrix (in the presence of valinomycin, the potassium carrier), as the matrix contains $0.1 \mathrm{M} \mathrm{K}^{+}[20,21,27]$. The efflux of the $\mathrm{K}^{+}$positive ion is equivalent to the influx of the negative one $\left(\mathrm{Cl}^{-}\right)$since, in both situations, the positive-inside potential diminishes, and an acidification of up to a $\mathrm{pH}$ of 4.2 occurs. In this condition, and in steady state ( $\mathrm{pH} 4.2)$, the addition of the aluminum induces the same rapid alkalinization as that obtained using a chloride medium, thus demonstrating that the presence of the chloride channel is of no significance.

- Uncoupling effect. An uncoupler is a particular type of chemical compound which enhances proton permeability. The uncoupler must be a weak acid (or a weak base), such as, for example, 2,4 dinitrophenol (DNP), which is the most common uncoupler. The DNP permeates the lysosomes as a phenate ion and the driving force is the positive-inside potential [20].

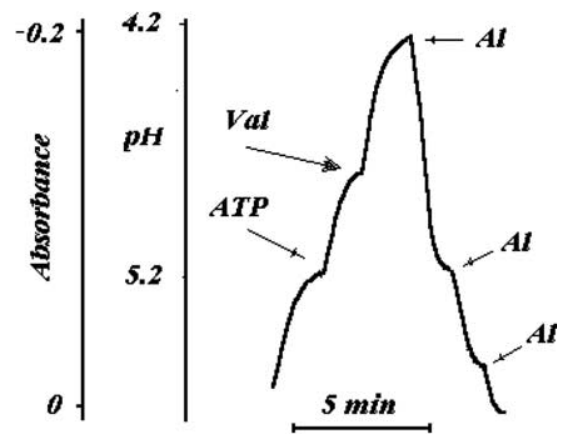

Fig. 5. Acidification of lysosomes in a chloride-free medium. Medium composition: $80 \mathrm{mM} \mathrm{K} \mathrm{SO}_{4}, 10 \mathrm{mM}$ Hepes-Mops pH 7.4, $10 \mathrm{mM}$ $\mathrm{MgSO}_{4}$. Lysosomes: $0.2 \mathrm{mg} / \mathrm{ml}$. When indicated, Valinomycin (Val) 10 $\mu \mathrm{M}, \mathrm{ATP}, 2 \mathrm{mM}$ and successive additions of $80 \mu \mathrm{M} \mathrm{Al}$ (in the form of lactate) were added.
Once inside, the DNP is extruded in the form of an electroneutral, undissociated DNP compound, as the $\mathrm{pH}$ is acid inside. During this cyclic mechanism [28], each cycle gives rise to a proton extrusion. Fig. 6(a) shows the effect induced by the DNP, which is similar to that obtained by the addition of aluminum (Fig. 2). The experiment in Fig. 6(a) seems to indicate that aluminum behaves as an uncoupler, and that a possible uncoupling mechanism could be the uptake of $\mathrm{Al}(\mathrm{OH})_{3}$ (since the $\mathrm{pH}$ is acid inside) and the extrusion of $\mathrm{Al}(\mathrm{OH})_{2}^{+}$(since the potential is positive inside).

- Weak base effect. Weak permeant bases induce alkalinization in lysosomes (Fig. 6(b)). In this regard, the interpretative mechanism is that the weak base enters the lysosomes as an electroneutral compound, and is accumulated in the matrix, since the $\mathrm{pH}$ is acid inside. This accumulation is not followed by an extrusion in the form of a charged ion, as occurs in the case of uncouplers (weak base uncouplers). Therefore, as the mechanism is not cyclic, the dose of weak bases necessary to induce alkalinization is higher than that needed to obtain the same effect with the addition of uncouplers [23]. In the case of DNP, the dose necessary to induce a rapid alkalinization is about 100 $\mu \mathrm{M}$, in that of ammonia, the concentration required is $10 \mathrm{mM}$.

Therefore, it can be said that both types of behaviour, that is, the uncoupling and the weak base effect, are possible in the case of aluminum, with regard to the rapid alkalinization process. However, as the concentration of aluminum is in the order of magnitude of micromolar, it would seem, judging from the results of the latest experiments, that aluminum is a protonophor or uncoupler, rather than a weak base.

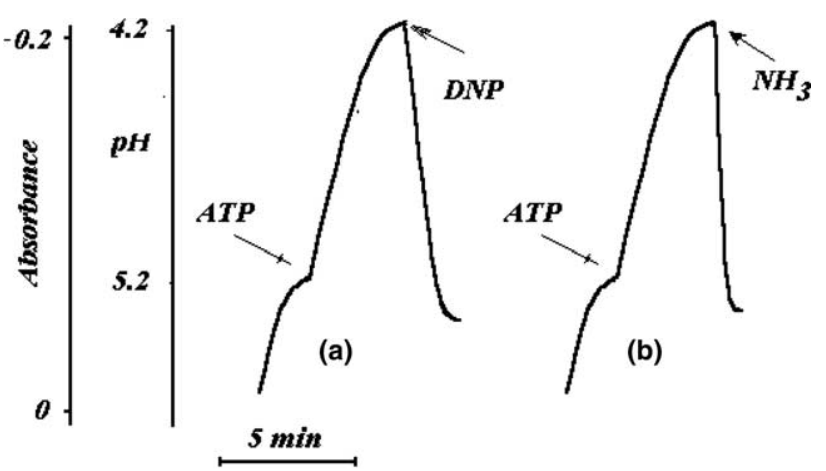

Fig. 6. The uncoupler, DNP and the weak base, ammonia, induce a rapid alkalinization in lysosomes. Both the medium used and conditions were the same as in Fig. 2. The addition of $100 \mu \mathrm{M}$ DNP (a) or 10 $\mathrm{mM}$ ammonia (in the form of ammonium chloride) (b) in steady state induce a rapid alkalinization in energized lysosomes. The addition of ammonia does not change the $\mathrm{pH}$ in the resuspending medium. Doses of ammonia below millimolar do not induce alkalinization (not shown). 


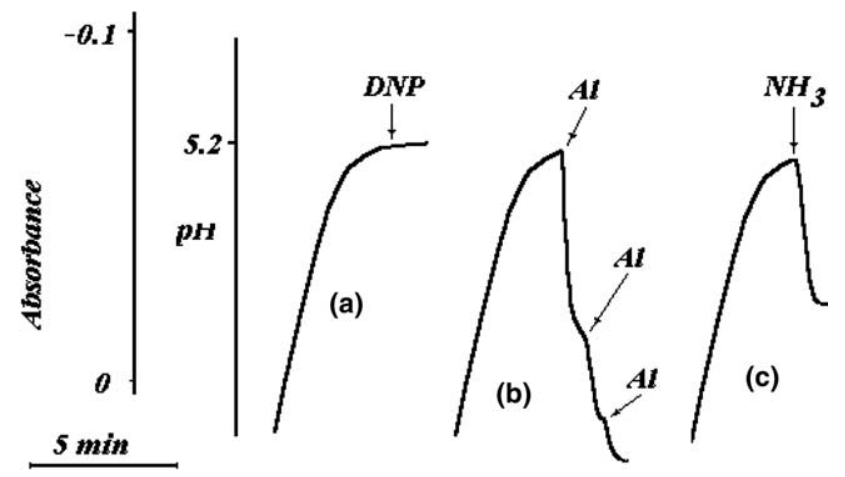

Fig. 7. The effects of the uncouplers DNP, aluminum or of the weak base, ammonia, in non-energized lysosomes. Both the medium used and the conditions were the same as in Fig. 2 before the ATP addition. In steady state, the addition of DNP does not induce alkalinization (a), while repeated addition of $80 \mu \mathrm{M} \mathrm{Al}$ (lactate) $)_{3}$ does induce alkalinization (b) as is also true in the case of the addition of ammonia (ammonium chloride) $10 \mathrm{mM}$ (c).

Some experiments using non-energized lysosomes were performed, in order to clarify the above observations.

The internal $\mathrm{pH}$ in lysosomes, before ATP addition, is 5.2, as already mentioned [19]. The addition of an uncoupler under these conditions does not induce any alkalinization (Fig. 7(a)), since the action of an uncoupler requires the presence of a membrane potential, while the addition of aluminum induces a rapid alkalinization (Fig. 7(b)) similar to that produced after the addition of ammonia (Fig. 7(c)). This type of behaviour (which is not ATPase-dependent as the lysosomes are not energized) should exclude the possibility that aluminum is an uncoupler. Therefore, the only possible deduction that can be made is that aluminum enters the lysosomes in the form of an $\mathrm{Al}(\mathrm{OH})_{3}$ compound.

However, in the experiments of Fig. 7 (non-energized lysosomes), the potential is absent. This fact cannot exclude the possibility that in the presence of a potential (energized lysosomes) $\mathrm{Al}(\mathrm{OH})_{2}^{+}$is extruded, thus giving rise to a cyclic (uncoupling) mechanism. At the present time, therefore, both mechanisms are possible, since, whereas it is evident that $\mathrm{Al}(\mathrm{OH})_{3}$ enters like a weak base, the driving force being the internal acidic $\mathrm{pH}$, this cannot exclude the possibility (even if not demonstrated), that, once inside, the cation $\mathrm{Al}(\mathrm{OH})_{2}^{+}$is extruded. In this case it results to be an uncoupling mechanism.

This last mechanism, being cyclic, can explain why the dose of aluminum necessary for the alkalinization process is more than an order of magnitude lower than that needed for a weak base such as ammonia.

Both interpretations are different from the one proposed by Zatta et al. [12] which asserts that the inhibition of the alkalinization induced by aluminum is due to an inhibition of the ATPase. This inhibition has been verified (Fig. 3), but it is not the cause of the rapid alkalinization rate observed after addition of aluminum. The interpretation of Zatta et al. [12], could be explained by the fact that the addition of aluminum before ATP does not allow to distinguish among the various possible mechanisms.

Another point to explain is why the addition of aluminum induces a more marked alkalinization than it does in the case of ammonia. A possible explanation is that $\mathrm{Al}(\mathrm{OH})_{3}$ penetrates the lysosomes and once inside, $\mathrm{Al}^{3+}$ binds closely to internal ligands, thus favouring a further uptake of $\mathrm{Al}(\mathrm{OH})_{3}$ and a consequent alkalinization. A possible target for internal binding are the phosphate groups, since the affinity of $\mathrm{Al}^{3+}$ is about three orders of magnitude higher than that of $\mathrm{Mg}^{++}$[29] which strongly binds the phosphate groups in the membranes.

The above experiments suggest that the permeation properties of $\mathrm{Al}(\mathrm{OH})_{3}$ which were evidenced by means of the alkalinization experiments performed on nonenergized lysosomes, relate to the fact that this compound behaves as a weak (permeant) base. Like the weak bases, the undissociated $\mathrm{Al}(\mathrm{OH})_{3}$ compound, even if present in small amounts, is a permeant compound, which crosses the membrane and binds to the internal ligands in the lysosomes. This uptake mechanism does not exclude the possibility that aluminum, in energized lysosomes, once inside, is extruded as monovalent cation $\mathrm{Al}(\mathrm{OH})_{2}^{+}$from the positive potential, thus giving rise to a cyclic mechanism similar to that of uncouplers.

The crucial point, however, is that the electroneutral $\mathrm{Al}(\mathrm{OH})_{3}$ compound, even if present in small concentrations, crosses the biological membranes. This behaviour can explain the presence of aluminum inside the cells without postulating the utilization of carrier transport systems.

\section{References}

[1] A.C. Alfrey, G.R. LeGendre, W.D. Kaehny, Engl. J. Med. 294 (1976) 184-188.

[2] A.C. Alfrey, Life Chem. Rep. 11 (1994) 197-205.

[3] C.V. Swegert, K.R. Dave, S.S. Katyare, Mech. Ageing Dev. 112 (1999) 27-42.

[4] T.L. Petit, G.B. Biederman, P.A. McMullen, Exp. Neurol. 67 (1980) 152-162.

[5] K.S. Rao, G.V. Rao, Mol. Cell. Biochem. 137 (1994) 57-60.

[6] H.M. Wisniewski, J.A. Sturman, J.W. Shek, Ann. Neurol. 8 (1980) 479-490.

[7] H.M. Wisniewski, K. Iqbal, Neurotoxicology 1 (1980) 121-129.

[8] C.D. Hewitt, M.M. Herman, M.B. Lopes, J. Savory, M.R. Wills, Neuropathol. Appl. Neurobiol. 17 (1991) 47-60.

[9] A. Toninello, G. Clari, M. Mancon, G. Tognon, P. Zatta, J. Biol. Inorg. Chem. 5 (2000) 612-623.

[10] M. Colombini, J. Membr. Biol. 111 (1989) 103-111. 
[11] T. Mirzabekov, C. Ballarin, M. Nicolini, P. Zatta, M.C. Sorgato, J. Membr. Biol. 133 (1993) 129-143.

[12] P. Zatta, A. Taylor, P. Zambenedetti, R. Milacic, P. Dell'Antone, Life Sci. 66 (2000) 2261-2266.

[13] R.B. Martin, J. Inorg. Biochem. 28 (1986) 181-187.

[14] R.B. Martin, Metal Ions Biol. Syst. 24 (1988) 1-9.

[15] R.B. Martin, Clin. Chem. 32 (1986) 1797-1806.

[16] E. Marklung, L. Ohman, Acta Chem. Scand. 44 (1990) 353-357.

[17] P.L. Savant, S. Shibo, U.S. Kumta, A.L. Tappel, Biochim. Biophys. Acta 85 (1964) 82-92.

[18] O.H. Lowry, N.Y. Rosemberg, D.L. Farr, R.S. Randall, J. Biol. Chem. 193 (1951) 265-275.

[19] Y. Muriyama, M. Maeda, M. Futai, FEBS Lett. 302 (1992) 18-20.

[20] S. Ohkuma, Y. Moriyama, T. Takano, J. Biochem. 94 (1983) 1935-1943.
[21] I. Mellman, R. Fuchs, A. Helenius, Ann. Rev. Biochem. 55 (1986) 663-700.

[22] P.O. Segeln, Methods Enzymol. 96 (1993) 737-766.

[23] P. Dell'Antone, M. Bragadin, P. Zatta, Biochim. Biophys. Acta 1270 (1995) 137-141.

[24] S. Ohkuma, B. Poole, J. Cell Biol. 90 (1981) 656-664.

[25] P. Dell'Antone, R. Colonna, G.F. Azzone, Eur. J. Biochem. 24 (1972) 553-576.

[26] E.J. Bowman, A. Siebers, K. Altendorf, Proc. Natl. Acad. Sci. USA 85 (1988) 7972-7975.

[27] P. Dell'Antone, Biochem. Biophys. Res. Commun. 86 (1979) 180 189.

[28] P. Mitchell, Science 206 (1979) 1148-1159.

[29] M. Nicolini, P. Zatta, B. Corain in Aluminum in Chemistry Biology and Medicine, Cortina International-Verona Raven Press, New York, 1991. 